\title{
Oxidative Transformation of Fluoroquinolone Antibacterial Agents and Structurally Related Amines by Manganese Dioxide
}

\author{
Huichun Zhang and Ching-Hua Huang* \\ School of Civil and Environmental Engineering, Georgia Institute of Technology \\ Atlanta, Georgia 30332 \\ *Corresponding author phone: 404-894-7694; fax: 404-894-8266; e-mail: \\ ching-hua.huang@ce.gatech.edu.
}

Table S1. LC-ESI-MS fragments of ciprofloxacin (CIP) and its oxidation products.

Table S2. LC-ESI-MS fragments of enrofloxacin (ENR) and its oxidation products.

Table S3. LC-ESI-MS fragments of norfloxacin (NOR) and its oxidation products.

Table S4. LC-ESI-MS fragments of ofloxacin (OFL) and its oxidation products.

Table S5. LC-ESI-MS fragments of lomefloxacin (LOM) and its oxidation products.

Table S6. LC-ESI-MS fragments of pipemidic acid (PIP) and its oxidation products.

Table S7. Product distribution (\%) of FQs in reaction with $\mathrm{MnO}_{2}$.

Table S8. LC-ESI-MS fragments of 1-phenylpiperazine (PP) and its oxidation products.

Table S9. LC-ESI-MS fragments of 1-phenylmorpholine (PM) and its oxidation products.

Figure S1. Effect of CIP and $\mathrm{MnO}_{2}$ loadings on the initial reaction rate $\left(\mathrm{pH} 5,22{ }^{\circ} \mathrm{C}\right)$.

Figure S2. FTIR spectrum of the mixture of PP oxidation products. 
Table S1. LC-ESI-MS fragments of ciprofloxacin (CIP) and its oxidation products.

\begin{tabular}{|c|c|c|c|c|c|c|c|c|c|c|c|c|c|c|c|}
\hline & & \multicolumn{2}{|c|}{$\begin{array}{c}\text { CIP } \\
\mathrm{M}\end{array}$} & \multicolumn{2}{|c|}{$\mathrm{M}+2$} & \multicolumn{2}{|c|}{ M-26 } & \multicolumn{2}{|c|}{$\mathrm{M}+30$} & \multicolumn{2}{|c|}{$\mathrm{M}+2$} & \multicolumn{2}{|c|}{ M-69 } & \multicolumn{2}{|c|}{$\mathrm{M}+32$} \\
\hline \multirow[t]{2}{*}{ RT (min) } & & \multicolumn{2}{|c|}{12.78} & \multicolumn{2}{|c|}{9.93} & \multicolumn{2}{|c|}{13.49} & \multicolumn{2}{|c|}{18.75} & \multicolumn{2}{|c|}{21.89} & \multicolumn{2}{|c|}{23.69} & \multicolumn{2}{|c|}{14.76} \\
\hline & & $\mathrm{m} / \mathrm{z}$ & int. & $\mathrm{m} / \mathrm{z}$ & int. & $\mathrm{m} / \mathrm{z}$ & int. & $\mathrm{m} / \mathrm{z}$ & int. & $\mathrm{m} / \mathrm{z}$ & int. & $\mathrm{m} / \mathrm{z}$ & int. & $\mathrm{m} / \mathrm{z}$ & int. \\
\hline$[\mathrm{MH}]^{+^{\bullet}}$ & 0 & 332 & 100 & 334 & 65 & 306 & 30 & 362 & 45 & 334 & 50 & 263 & 55 & 364 & 80 \\
\hline$[\mathrm{M}+\mathrm{Na}]^{+^{\circ}}$ & +22 & 354 & 6 & 356 & 10 & 328 & 10 & 384 & 15 & - & - & 285 & 8 & 386 & 8 \\
\hline$\left[\mathrm{MH}-\mathrm{H}_{2} \mathrm{O}\right]^{+}$ & -18 & 314 & 98 & 316 & 100 & 288 & 100 & 344 & 100 & 316 & 100 & 245 & 100 & 346 & 100 \\
\hline$\left[\mathrm{MH}-\mathrm{H}_{2} \mathrm{O}-\mathrm{HF}\right]^{{ }^{\bullet}}$ & -38 & - & - & - & - & 268 & 5 & - & - & - & - & - & - & - & - \\
\hline$\left[\mathrm{MH}-\mathrm{CO}_{2}\right]^{+^{\bullet}}$ & -44 & 288 & 60 & - & - & - & - & - & - & - & - & - & - & - & - \\
\hline$\left[\mathrm{MH}-\mathrm{H}_{2} \mathrm{O}-\mathrm{CO}\right]^{+^{\bullet}}$ & -46 & - & - & - & - & - & - & 316 & 10 & 288 & 8 & 217 & 5 & - & - \\
\hline$\left[\mathrm{MH}-\mathrm{H}_{2} \mathrm{O}-\nabla\right]^{+^{\bullet}}$ & -58 & 274 & 6 & - & - & - & - & - & - & - & - & - & - & 306 & 10 \\
\hline$\left[\mathrm{MH}-\mathrm{H}_{2} \mathrm{O}-\mathrm{C}_{2} \mathrm{H}_{5} \mathrm{~N}\right]^{+^{\bullet}}$ & -61 & - & - & 273 & 60 & 245 & 12 & - & - & - & - & - & - & - & - \\
\hline$\left[\mathrm{MH}-\mathrm{H}_{2} \mathrm{O}-\mathrm{CO}_{2}\right]^{+^{\bullet}}$ & -62 & - & - & - & - & - & - & 300 & 8 & - & - & - & - & - & - \\
\hline$\left[\mathrm{MH}-\mathrm{HF}-\mathrm{CO}_{2}\right]^{\bullet^{\bullet}}$ & -64 & - & - & - & - & - & - & - & - & 270 & 15 & - & - & - & - \\
\hline$\left[\mathrm{MH}-\mathrm{H}_{2} \mathrm{O}-2 \mathrm{CO}\right]^{+^{\bullet}}$ & -74 & - & - & - & - & - & - & 288 & 10 & - & - & - & - & - & - \\
\hline$\left[\mathrm{MH}-2 \mathrm{H}_{2} \mathrm{O}-\nabla\right]^{+^{\bullet}}$ & -76 & - & - & - & - & - & - & - & - & - & - & - & - & 288 & 6 \\
\hline$\left[\mathrm{MH}-\mathrm{H}_{2} \mathrm{O}-\mathrm{HF}-\mathrm{C}_{2} \mathrm{H}_{5} \mathrm{~N}\right]^{{ }^{\bullet}}$ & -81 & - & - & - & - & - & - & - & - & - & - & - & - & - & - \\
\hline$\left[\mathrm{MH}-\mathrm{CO}_{2}-\mathrm{C}_{2} \mathrm{H}_{5} \mathrm{~N}\right]^{+^{\bullet}}$ & -87 & 245 & 25 & - & - & - & - & - & - & - & - & - & - & - & - \\
\hline$\left[\mathrm{MH}-\mathrm{H}_{2} \mathrm{O}-\mathrm{CO}-\mathrm{C}_{2} \mathrm{H}_{5} \mathrm{~N}\right]^{{ }^{\bullet}}$ & -89 & - & - & - & - & 217 & 5 & 273 & 13 & - & - & - & - & - & - \\
\hline$\left[\mathrm{MH}-\mathrm{H}_{2} \mathrm{O}-\nabla-\mathrm{C}_{2} \mathrm{H}_{5} \mathrm{~N}\right]^{+^{\bullet}}$ & -101 & 231 & 20 & - & - & - & - & - & - & - & - & - & - & - & - \\
\hline$\left[\mathrm{MH}-\mathrm{HF}-\nabla-\mathrm{C}_{2} \mathrm{H}_{5} \mathrm{~N}\right]^{{ }^{\bullet}}$ & -103 & - & - & 231 & 25 & 203 & 6 & 259 & 8 & - & - & - & - & - & - \\
\hline$\left[\mathrm{MH}-\mathrm{H}_{2} \mathrm{O}-2 \mathrm{CO}-\mathrm{C}_{2} \mathrm{H}_{5} \mathrm{~N}\right]^{+^{\bullet}}$ & -117 & - & - & - & - & - & - & - & - & 217 & 10 & - & - & - & - \\
\hline$\left[\mathrm{MH}-\mathrm{H}_{2} \mathrm{O}-\mathrm{CO}-\nabla-\mathrm{C}_{2} \mathrm{H}_{5} \mathrm{~N}\right]^{+^{\bullet}}$ & -129 & 203 & 3 & - & - & - & - & - & - & - & - & - & - & - & - \\
\hline$\left[\mathrm{MH}-\mathrm{H}_{2} \mathrm{O}-\mathrm{CO}-\mathrm{CO}_{2}-\mathrm{C}_{2} \mathrm{H}_{5} \mathrm{~N}\right]^{+^{\bullet}}$ & -133 & - & - & - & - & - & - & - & - & - & - & - & - & 231 & 10 \\
\hline$\left[\mathrm{MH}-\mathrm{H}_{2} \mathrm{O}-3 \mathrm{CO}-\mathrm{C}_{2} \mathrm{H}_{5} \mathrm{~N}\right]^{+^{\bullet}}$ & -145 & - & - & - & - & - & - & 217 & 6 & - & - & - & - & - & - \\
\hline$\left[\mathrm{MH}-2 \mathrm{H}_{2} \mathrm{O}-\mathrm{HF}-\mathrm{C}_{2} \mathrm{H}_{5} \mathrm{~N}-\nabla\right]^{+^{\bullet}}$ & -147 & - & - & - & - & - & - & - & - & - & - & - & - & 217 & 8 \\
\hline$\left[\mathrm{MH}-\mathrm{H}_{2} \mathrm{O}-2 \mathrm{CO}-\mathrm{CO}_{2}-\mathrm{C}_{2} \mathrm{H}_{5} \mathrm{~N}\right]^{+}$ & -161 & - & - & - & - & - & - & - & - & - & - & - & - & 203 & 10 \\
\hline
\end{tabular}

$\mathrm{RT}=\mathrm{LC}$ retention time; int. $=\mathrm{MS}$ intensity $(\%) ; \nabla=$ cyclic propyl ring. 
Table S2. LC-ESI-MS fragments of enrofloxacin (ENR) and its oxidation products.

\begin{tabular}{|c|c|c|c|c|c|c|c|c|c|c|c|c|c|c|c|c|c|}
\hline \multirow{3}{*}{ RT (min) } & & \multicolumn{2}{|c|}{$\begin{array}{c}\text { ENR } \\
\mathrm{M}\end{array}$} & \multicolumn{2}{|c|}{$\mathrm{M}+2$} & \multicolumn{2}{|c|}{ M-26 } & \multicolumn{2}{|c|}{$\begin{array}{c}\text { CIP } \\
\text { analog }\end{array}$} & \multicolumn{2}{|c|}{$\mathrm{M}+30$} & \multicolumn{2}{|c|}{ M-69* } & \multicolumn{2}{|c|}{$\mathrm{M}+2$} & \multicolumn{2}{|c|}{$M+16$} \\
\hline & & \multicolumn{2}{|c|}{17.289} & \multicolumn{2}{|c|}{13.081} & \multicolumn{2}{|c|}{14.168} & \multicolumn{2}{|c|}{14.814} & \multicolumn{2}{|c|}{21.626} & \multicolumn{2}{|c|}{23.452} & \multicolumn{2}{|c|}{29.161} & \multicolumn{2}{|c|}{15.308} \\
\hline & & $\mathrm{m} / \mathrm{z}$ & int. & $\mathrm{m} / \mathrm{z}$ & int. & $\mathrm{m} / \mathrm{z}$ & int. & $\mathrm{m} / \mathrm{z}$ & int. & $\mathrm{m} / \mathrm{z}$ & int. & $\mathrm{m} / \mathrm{z}$ & int. & $\mathrm{m} / \mathrm{z}$ & int. & $\mathrm{m} / \mathrm{z}$ & int. \\
\hline$[\mathrm{MH}]^{+^{\bullet}}$ & 0 & 360 & 100 & 362 & 60 & 334 & 68 & 332 & 100 & 390 & 98 & 263 & 40 & 362 & 60 & 376 & 30 \\
\hline$[\mathrm{M}+\mathrm{Na}]^{+^{\bullet}}$ & +22 & 382 & 10 & - & - & 356 & 8 & 354 & 10 & 412 & 6 & 285 & 10 & - & - & 398 & 10 \\
\hline$\left[\mathrm{MH}-\mathrm{H}_{2} \mathrm{O}\right]^{+^{\bullet}}$ & -18 & 342 & 55 & 344 & 55 & 316 & 100 & 314 & 70 & 372 & 100 & 245 & 100 & 344 & 55 & 358 & 100 \\
\hline$\left[\mathrm{MH}-\mathrm{H}_{2} \mathrm{O}-\mathrm{HF}\right]^{{ }^{\bullet}}$ & -38 & - & - & - & - & 296 & 10 & - & - & - & - & - & - & - & - & - & - \\
\hline$\left[\mathrm{MH}-\mathrm{CO}_{2}\right]^{+^{\bullet}}$ & -44 & 316 & 80 & - & - & - & - & 288 & 62 & 346 & 45 & - & - & - & - & 332 & 10 \\
\hline$\left[\mathrm{MH}-\mathrm{CO}-\mathrm{NH}_{3}\right]^{{ }^{\circ}}$ & -45 & - & - & 317 & 55 & - & - & - & - & - & - & - & - & 317 & 55 & - & - \\
\hline$\left[\mathrm{MH}-\mathrm{H}_{2} \mathrm{O}-\nabla\right]^{{ }^{\circ}}$ & -58 & 302 & 5 & - & - & - & - & - & - & - & - & - & - & - & - & - & - \\
\hline$\left[\mathrm{MH}-\mathrm{H}_{2} \mathrm{O}-\mathrm{CO}\right]^{{ }^{*}}$ & -46 & - & - & - & - & - & - & - & - & - & - & 217 & 8 & - & - & - & - \\
\hline$[\mathrm{MH}-\mathrm{HF}-\nabla]^{+^{\bullet}}$ & -60 & - & - & - & - & - & - & - & - & - & - & - & - & - & - & 316 & 35 \\
\hline$\left[\mathrm{MH}-\mathrm{H}_{2} \mathrm{O}-\mathrm{CO}_{2}\right]^{+^{\circ}}$ & -62 & - & - & - & - & - & - & - & - & 328 & 88 & - & - & - & - & 314 & 58 \\
\hline$\left[\mathrm{MH}-\mathrm{HF}-\mathrm{CO}_{2}\right]^{+^{\bullet}}$ & -64 & - & - & 298 & 45 & - & - & 268 & 20 & - & - & - & - & 298 & 45 & - & - \\
\hline$\left[\mathrm{MH}-\mathrm{C}_{4} \mathrm{H}_{7} \mathrm{~N}\right]^{+}$ & -69 & - & - & - & - & - & - & 263 & 5 & - & - & - & - & - & - & - & - \\
\hline$\left[\mathrm{MH}-\mathrm{CO}-\mathrm{C}_{2} \mathrm{H}_{5} \mathrm{~N}\right]^{+}$ & -71 & - & - & - & - & 263 & 10 & - & - & - & - & - & - & - & - & - & - \\
\hline$\left[\mathrm{MH}-\mathrm{CO}_{2}-\mathrm{C}_{2} \mathrm{H}_{5} \mathrm{~N}\right]^{+^{\bullet}}$ & -87 & - & - & - & - & - & - & 245 & 55 & - & - & - & - & - & - & - & - \\
\hline$[\mathrm{MH}-\mathrm{HF}-\mathrm{CO}-\nabla]^{+^{\bullet}}$ & -88 & - & - & - & - & - & - & - & - & - & - & - & - & - & - & 288 & 10 \\
\hline$\left[\mathrm{MH}-\mathrm{H}_{2} \mathrm{O}-\mathrm{CO}-\mathrm{C}_{2} \mathrm{H}_{5} \mathrm{~N}\right]^{+^{\bullet}}$ & -89 & - & - & 273 & 100 & 245 & 12 & - & - & 301 & 43 & - & - & 273 & 100 & - & - \\
\hline$\left[\mathrm{MH}-\mathrm{CO}-\mathrm{CO}_{2}-\mathrm{C}_{2} \mathrm{H}_{5} \mathrm{~N}\right]^{+^{\bullet}}$ & -115 & 245 & 18 & - & - & 219 & 8 & - & - & - & - & - & - & - & - & - & - \\
\hline$\left[\mathrm{MH}-\mathrm{H}_{2} \mathrm{O}-2 \mathrm{CO}-\mathrm{C}_{2} \mathrm{H}_{5} \mathrm{~N}\right]^{{ }^{\bullet}}$ & -117 & - & - & 245 & 28 & - & - & - & - & - & - & - & - & 245 & 28 & - & - \\
\hline$\left[\mathrm{MH}-\mathrm{HF}-2 \mathrm{CO}-\mathrm{C}_{2} \mathrm{H}_{5} \mathrm{~N}\right]^{+}$ & -119 & - & - & - & - & - & - & - & - & - & - & - & - & - & - & 257 & 20 \\
\hline$\left[\mathrm{MH}-\mathrm{H}_{2} \mathrm{O}-\mathrm{CO}-\nabla-\mathrm{C}_{2} \mathrm{H}_{5} \mathrm{~N}\right]^{{ }^{\bullet}}$ & -129 & 231 & 8 & - & - & - & - & - & - & - & - & - & - & - & - & - & - \\
\hline$\left[\mathrm{MH}-\mathrm{HF}-\mathrm{CO}-\nabla-\mathrm{C}_{2} \mathrm{H}_{5} \mathrm{~N}\right]^{+^{\bullet}}$ & -131 & - & - & 231 & 5 & - & - & - & - & - & - & - & - & - & - & 245 & 6 \\
\hline$\left[\mathrm{MH}-\mathrm{H}_{2} \mathrm{O}-3 \mathrm{CO}-\mathrm{C}_{2} \mathrm{H}_{5} \mathrm{~N}\right]^{+^{*}}$ & -145 & - & - & 217 & 8 & - & - & - & - & - & - & - & - & 217 & 8 & - & - \\
\hline
\end{tabular}

$\mathrm{RT}=\mathrm{LC}$ retention time; int. $=\mathrm{MS}$ intensity $(\%) ; \nabla=$ cyclic propyl ring.

*: classified as the M-69 product because of its structural similarity to the M-69 product of CIP. 
Table S3. LC-ESI-MS fragments of norfloxacin (NOR) and its oxidation products.

\begin{tabular}{|c|c|c|c|c|c|c|c|c|c|c|c|c|c|c|c|c|c|}
\hline & & $\begin{array}{r}\mathrm{NO} \\
\mathrm{N} \\
\end{array}$ & & $\mathrm{M}$ & & $\mathrm{M}$ & & $\mathrm{M}-$ & -32 & & -30 & $\mathrm{M}$ & +2 & $\mathrm{M}$ & & & \\
\hline \multirow[t]{2}{*}{ RT (min) } & & \multicolumn{2}{|c|}{14.508} & \multicolumn{2}{|c|}{8.826} & \multicolumn{2}{|c|}{12.54} & \multicolumn{2}{|c|}{13.87} & \multicolumn{2}{|c|}{18.043} & \multicolumn{2}{|c|}{21.046} & \multicolumn{2}{|c|}{21.797} & \multicolumn{2}{|c|}{16.666} \\
\hline & & $\mathrm{m} / \mathrm{z}$ & int. & $\mathrm{m} / \mathrm{z}$ & int. & $\mathrm{m} / \mathrm{z}$ & int. & $\mathrm{m} / \mathrm{z}$ & int. & $\mathrm{m} / \mathrm{z}$ & int. & $\mathrm{m} / \mathrm{z}$ & int. & $\mathrm{m} / \mathrm{z}$ & int. & $\mathrm{m} / \mathrm{z}$ & int. \\
\hline$[\mathrm{MH}]^{+^{\bullet}}$ & 0 & 320 & 100 & 322 & 50 & 294 & 40 & 352 & 40 & 350 & 28 & 322 & 60 & 251 & 20 & 348 & 70 \\
\hline$[\mathrm{M}+\mathrm{Na}]^{+^{\bullet}}$ & +22 & 342 & 5 & - & - & 306 & 15 & - & - & 372 & 10 & 344 & 15 & 273 & 6 & 370 & 10 \\
\hline$\left[\mathrm{MH}-\mathrm{H}_{2} \mathrm{O}\right]^{+}$ & -18 & 302 & 53 & 304 & 100 & 276 & 100 & 334 & 100 & 332 & 100 & 304 & 100 & 233 & 100 & 330 & 15 \\
\hline$\left[\mathrm{MH}-\mathrm{H}_{2} \mathrm{O}-\mathrm{HF}\right]^{+^{\bullet}}$ & -38 & - & - & 284 & 28 & 256 & 40 & - & - & - & - & - & - & - & - & - & - \\
\hline$\left[\mathrm{MH}-\mathrm{C}_{2} \mathrm{H}_{5} \mathrm{~N}\right]^{+^{\bullet}}$ & -43 & - & - & - & - & 251 & 5 & - & - & - & - & 279 & 43 & - & - & - & - \\
\hline$\left[\mathrm{MH}-\mathrm{CO}_{2}\right]^{\bullet^{\bullet}}$ & -44 & 276 & 75 & - & - & - & - & - & - & - & - & - & - & - & - & - & - \\
\hline$\left[\mathrm{MH}-\mathrm{H}_{2} \mathrm{O}-\mathrm{CO}\right]^{+^{\bullet}}$ & -46 & - & - & - & - & - & - & - & - & 304 & 6 & - & - & 205 & 15 & 302 & 6 \\
\hline$\left[\mathrm{MH}-\mathrm{H}_{2} \mathrm{O}-\mathrm{C}_{2} \mathrm{H}_{5} \mathrm{~N}\right]^{{ }^{\bullet}}$ & -61 & - & - & 261 & 80 & 233 & 30 & - & - & - & - & 261 & 86 & - & - & - & - \\
\hline$\left[\mathrm{MH}-\mathrm{HF}-\mathrm{C}_{2} \mathrm{H}_{5} \mathrm{~N}\right]^{{ }^{\bullet}}$ & -63 & - & - & - & - & 231 & 5 & - & - & - & - & - & - & - & - & - & - \\
\hline$\left[\mathrm{MH}-\mathrm{CO}-\mathrm{C}_{2} \mathrm{H}_{5} \mathrm{~N}\right]^{+}$ & -71 & - & - & - & - & - & - & - & - & - & - & 251 & 30 & - & - & - & - \\
\hline$\left[\mathrm{MH}-\mathrm{HF}-\mathrm{CO}-\mathrm{C}_{2} \mathrm{H}_{5}\right]^{{ }^{\bullet}}$ & -76 & - & - & - & - & - & - & 276 & 15 & - & - & - & - & - & - & - & - \\
\hline$\left[\mathrm{MH}-\mathrm{CO}_{2}-\mathrm{C}_{2} \mathrm{H}_{5} \mathrm{~N}\right]^{+^{\bullet}}$ & -87 & 233 & 20 & - & - & - & - & - & - & - & - & - & - & - & - & - & - \\
\hline$\left[\mathrm{MH}-\mathrm{H}_{2} \mathrm{O}-\mathrm{CO}-\mathrm{C}_{2} \mathrm{H}_{5} \mathrm{~N}\right]^{+^{\bullet}}$ & -89 & - & - & 233 & 20 & - & - & - & - & - & - & 233 & 15 & - & - & - & - \\
\hline$\left[\mathrm{MH}-\mathrm{CO}-\mathrm{CO}_{2}-\mathrm{C}_{2} \mathrm{H}_{5} \mathrm{~N}\right]^{+}$ & -115 & 205 & 8 & - & - & - & - & - & - & - & - & - & - & - & - & - & - \\
\hline$\left[\mathrm{MH}-\mathrm{HF}-2 \mathrm{CO}-\mathrm{C}_{2} \mathrm{H}_{5} \mathrm{~N}\right]^{+^{\bullet}}$ & -119 & - & - & - & - & - & - & - & - & 231 & 6 & - & - & - & - & - & - \\
\hline$\left[\mathrm{MH}-\mathrm{HF}-3 \mathrm{CO}-\mathrm{C}_{2} \mathrm{H}_{5} \mathrm{~N}\right]^{+}$ & -147 & - & - & - & - & - & - & 205 & 10 & - & - & - & - & - & - & - & - \\
\hline
\end{tabular}

$\mathrm{RT}=\mathrm{LC}$ retention time; int. $=\mathrm{MS}$ intensity $(\%)$. 
Table S4. LC-ESI-MS fragments of ofloxacin (OFL) and its oxidation products.

\begin{tabular}{|c|c|c|c|c|c|c|c|c|c|c|c|c|c|c|c|c|c|c|c|}
\hline & & \multicolumn{2}{|c|}{$\begin{array}{c}\text { OFL } \\
\mathrm{M}\end{array}$} & \multicolumn{2}{|c|}{$M+16$} & \multicolumn{2}{|c|}{ M-26 } & \multicolumn{2}{|c|}{$\mathrm{M}+30$} & \multicolumn{2}{|c|}{ M-69* } & \multicolumn{2}{|c|}{$\mathrm{M}+2$} & \multicolumn{2}{|c|}{$\mathrm{M}+2$} & \multicolumn{2}{|c|}{ ? } & \multicolumn{2}{|c|}{ ? } \\
\hline \multirow[t]{2}{*}{$\mathrm{RT}(\min )$} & & \multicolumn{2}{|c|}{14.163} & \multicolumn{2}{|c|}{11.108} & \multicolumn{2}{|c|}{13.464} & \multicolumn{2}{|c|}{20.831} & \multicolumn{2}{|c|}{22.994} & \multicolumn{2}{|c|}{24.419} & \multicolumn{2}{|c|}{25.116} & \multicolumn{2}{|c|}{7.627} & \multicolumn{2}{|c|}{17.529} \\
\hline & & $\mathrm{m} / \mathrm{z}$ & int. & $\mathrm{m} / \mathrm{z}$ & int. & $\mathrm{m} / \mathrm{z}$ & int. & $\mathrm{m} / \mathrm{z}$ & int. & $\mathrm{m} / \mathrm{z}$ & int. & $\mathrm{m} / \mathrm{z}$ & int. & $\mathrm{m} / \mathrm{z}$ & int. & $\mathrm{m} / \mathrm{z}$ & int. & $\mathrm{m} / \mathrm{z}$ & int. \\
\hline$[\mathrm{MH}]^{+^{\bullet}}$ & 0 & 362 & 95 & 378 & 13 & 336 & 86 & 392 & 45 & 279 & 28 & 364 & 100 & 364 & 100 & 314 & 100 & 298 & 25 \\
\hline$[\mathrm{M}+\mathrm{Na}]^{+^{\bullet}}$ & +22 & 384 & 5 & - & - & 358 & 15 & 414 & 5 & 301 & 6 & 386 & 12 & 386 & 20 & 336 & 10 & 320 & 8 \\
\hline$\left[\mathrm{MH}-\mathrm{H}_{2} \mathrm{O}\right]^{+}$ & -18 & 344 & 18 & 360 & 100 & 318 & 10 & 374 & 100 & 261 & 100 & 346 & 85 & 346 & 20 & 296 & 70 & 280 & 65 \\
\hline$\left[\mathrm{MH}-\mathrm{H}_{2} \mathrm{O}-\mathrm{HF}\right]^{+^{\bullet}}$ & -38 & - & - & - & - & 298 & 40 & - & - & - & - & - & - & - & - & - & - & - & - \\
\hline$\left[\mathrm{MH}-\mathrm{CO}_{2}\right]^{{ }^{\bullet}}$ & -44 & 318 & 100 & - & - & - & - & - & - & - & - & - & - & - & - & - & - & - & - \\
\hline$\left[\mathrm{MH}-\mathrm{H}_{2} \mathrm{O}-\mathrm{CO}\right]^{{ }^{\bullet}}$ & -46 & - & - & - & - & - & - & 346 & 5 & - & - & 318 & 10 & - & - & 268 & 8 & - & - \\
\hline$\left[\mathrm{MH}-\mathrm{C}_{3} \mathrm{H}_{7} \mathrm{~N}\right]^{+}$ & -57 & - & - & - & - & 279 & 43 & - & - & - & - & - & - & - & - & - & - & - & - \\
\hline$\left[\mathrm{MH}-\mathrm{H}_{2} \mathrm{O}-\mathrm{C}_{3} \mathrm{H}_{4}\right]^{+^{\bullet}}$ & -58 & - & - & - & - & - & - & - & - & - & - & - & - & - & - & - & - & 240 & 45 \\
\hline$\left[\mathrm{MH}-\mathrm{H}_{2} \mathrm{O}-\mathrm{CO}_{2}\right]^{+^{\bullet}}$ & -62 & - & - & 316 & 55 & - & - & - & - & - & - & - & - & - & - & - & - & - & - \\
\hline$\left[\mathrm{MH}-\mathrm{HF}-\mathrm{CO}_{2}\right]^{+^{\bullet}}$ & -64 & - & - & - & - & - & - & - & - & - & - & - & - & - & - & 250 & 23 & - & - \\
\hline$\left[\mathrm{MH}-\mathrm{H}_{2} \mathrm{O}-\mathrm{C}_{3} \mathrm{H}_{7} \mathrm{~N}\right]^{+}$ & -75 & - & - & - & - & 261 & 100 & - & - & - & - & - & - & - & - & - & - & - & - \\
\hline$\left[\mathrm{MH}-\mathrm{CO}_{2}-\mathrm{C}_{3} \mathrm{H}_{4}\right]^{+^{\bullet}}$ & -84 & - & - & - & - & - & - & - & - & - & - & 280 & 15 & - & - & - & - & - & - \\
\hline$\left[\mathrm{MH}-\mathrm{CO}_{2}-\mathrm{C}_{3} \mathrm{H}_{7} \mathrm{~N}\right]^{+^{\bullet}}$ & -101 & 261 & 40 & - & - & 235 & 15 & - & - & - & - & 263 & 10 & 263 & 8 & - & - & - & - \\
\hline$\left[\mathrm{MH}-\mathrm{H}_{2} \mathrm{O}-\mathrm{CO}_{2}-\mathrm{C}_{3} \mathrm{H}_{4}\right]^{+^{\bullet}}$ & -102 & - & - & - & - & - & - & - & - & - & - & 262 & 20 & - & - & - & - & - & - \\
\hline$\left[\mathrm{MH}-\mathrm{H}_{2} \mathrm{O}-\mathrm{CO}-\mathrm{C}_{3} \mathrm{H}_{7} \mathrm{~N}\right]^{+}$ & -103 & - & - & - & - & - & - & 289 & 8 & - & - & - & - & - & - & - & - & - & - \\
\hline$\left[\mathrm{MH}-\mathrm{CO}-\mathrm{CO}_{2}-\mathrm{C}_{3} \mathrm{H}_{4}-\mathrm{C}_{3} \mathrm{H}_{7} \mathrm{~N}\right]^{+^{\circ}}$ & -169 & - & - & - & - & - & - & 223 & 7 & - & - & - & - & - & - & - & - & - & - \\
\hline
\end{tabular}

$\mathrm{RT}=\mathrm{LC}$ retention time; int. = MS intensity (\%).

*: classified as the M-69 product because of its structural similarity to the M-69 product of CIP. 
Table S5. LC-ESI-MS fragments of lomefloxacin (LOM) and its oxidation products.

\begin{tabular}{|c|c|c|c|c|c|c|c|c|c|c|c|c|c|c|c|}
\hline \multirow{3}{*}{ RT (min) } & & \multicolumn{2}{|c|}{$\begin{array}{c}\text { LOM } \\
\mathrm{M} \\
\end{array}$} & \multicolumn{2}{|c|}{$\mathrm{M}+32$} & \multicolumn{2}{|c|}{$\mathrm{M}+2$} & \multicolumn{2}{|c|}{$M+16$} & \multicolumn{2}{|c|}{$M+16$} & \multicolumn{2}{|c|}{$\mathrm{M}+30$} & \multicolumn{2}{|c|}{ M-69* } \\
\hline & & \multicolumn{2}{|c|}{16.411} & \multicolumn{2}{|c|}{11.493} & \multicolumn{2}{|c|}{10.369} & \multicolumn{2}{|c|}{12.892} & \multicolumn{2}{|c|}{14.252} & \multicolumn{2}{|c|}{20.322} & \multicolumn{2}{|c|}{25.032} \\
\hline & & $\mathrm{m} / \mathrm{z}$ & int. & $\mathrm{m} / \mathrm{z}$ & int. & $\mathrm{m} / \mathrm{z}$ & int. & $\mathrm{m} / \mathrm{z}$ & int. & $\mathrm{m} / \mathrm{z}$ & int. & $\mathrm{m} / \mathrm{z}$ & int. & $\mathrm{m} / \mathrm{z}$ & int. \\
\hline$[\mathrm{MH}]^{+^{\bullet}}$ & 0 & 352 & 100 & 384 & 10 & 354 & 100 & 368 & 10 & 368 & 6 & 382 & 98 & 269 & 88 \\
\hline$[\mathrm{M}+\mathrm{Na}]^{+^{\bullet}}$ & +22 & - & - & - & - & - & - & - & - & - & - & 404 & 30 & 291 & 15 \\
\hline$\left[\mathrm{MH}-\mathrm{H}_{2} \mathrm{O}\right]^{+}$ & -18 & 334 & 27 & 366 & 25 & 336 & 50 & 350 & 45 & 350 & 100 & 364 & 100 & 251 & 100 \\
\hline$[\mathrm{MH}-\mathrm{CO}]^{+}$ & -28 & - & - & - & - & - & - & - & - & - & - & - & - & 241 & 40 \\
\hline$\left[\mathrm{MH}-2 \mathrm{H}_{2} \mathrm{O}\right]^{+^{\bullet}}$ & -36 & - & - & 348 & 40 & - & - & - & - & - & - & - & - & - & - \\
\hline$\left[\mathrm{MH}^{\left.-\mathrm{CO}_{2}\right]^{+}}\right.$ & -44 & 308 & 55 & - & - & - & - & - & - & - & - & - & - & - & - \\
\hline$\left[\mathrm{MH}-\mathrm{H}_{2} \mathrm{O}-\mathrm{CO}\right]^{+^{\bullet}}$ & -46 & - & - & - & - & - & - & - & - & - & - & 336 & 6 & 223 & 40 \\
\hline$\left[\mathrm{MH}-\mathrm{H}_{2} \mathrm{O}-\mathrm{CO}_{2}\right]^{+^{\bullet}}$ & -62 & - & - & - & - & - & - & 306 & 100 & 306 & 60 & - & - & - & - \\
\hline$\left[\mathrm{MH}-2 \mathrm{H}_{2} \mathrm{O}-2 \mathrm{HF}\right]^{+^{\bullet}}$ & -76 & - & - & 308 & 30 & - & - & - & - & - & - & - & - & - & - \\
\hline$\left[\mathrm{MH}-\mathrm{CO}_{2}-\mathrm{C}_{2} \mathrm{H}_{5} \mathrm{~N}\right]^{+}$ & -87 & 265 & 40 & - & - & - & - & - & - & - & - & - & - & - & - \\
\hline$\left[\mathrm{MH}-\mathrm{HF}-\mathrm{CO}-\mathrm{C}_{2} \mathrm{H}_{5} \mathrm{~N}\right]^{{ }^{\bullet}}$ & -91 & - & - & - & - & - & - & - & - & - & - & 291 & 10 & - & - \\
\hline$\left[\mathrm{MH}-\mathrm{HF}-\mathrm{CO}-\mathrm{CO}_{2}\right]^{{ }^{\bullet}}$ & -92 & - & - & - & - & - & - & 276 & 30 & - & - & - & - & - & - \\
\hline$\left[\mathrm{MH}-\mathrm{CO}_{2}-\mathrm{C}_{3} \mathrm{H}_{7} \mathrm{~N}\right]^{{ }^{\bullet}}$ & -101 & 251 & 11 & - & - & - & - & - & - & - & - & - & - & - & - \\
\hline$\left[\mathrm{MH}-\mathrm{H}_{2} \mathrm{O}-\mathrm{CO}_{2}-\mathrm{C}_{2} \mathrm{H}_{5} \mathrm{~N}\right]^{+^{\bullet}}$ & -105 & - & - & - & - & - & - & 263 & 45 & - & - & - & - & - & - \\
\hline$\left[\mathrm{MH}-\mathrm{CO}-\mathrm{CO}_{2}-\mathrm{C}_{2} \mathrm{H}_{5} \mathrm{~N}\right]^{{ }^{\bullet}}$ & -115 & 237 & 10 & - & - & - & - & - & - & - & - & - & - & - & - \\
\hline$\left[\mathrm{MH}-\mathrm{HF}-2 \mathrm{CO}-\mathrm{C}_{2} \mathrm{H}_{5} \mathrm{~N}\right]^{+^{\bullet}}$ & -119 & - & - & 265 & 100 & - & - & - & - & - & - & 263 & 6 & - & - \\
\hline
\end{tabular}

$\mathrm{RT}=$ LC Retention time; int. $=$ MS intensity (\%).

*: classified as the M-69 product because of its structural similarity to the M-69 product of CIP. 
Table S6. LC-ESI-MS fragments of pipemidic acid (PIP) and its oxidation products.

\begin{tabular}{|c|c|c|c|c|c|c|c|c|c|c|c|c|c|}
\hline \multirow{3}{*}{ RT (min) } & & \multicolumn{2}{|c|}{$\begin{array}{c}\text { PIP } \\
\mathrm{M} \\
\end{array}$} & \multicolumn{2}{|c|}{$M+16$} & \multicolumn{2}{|c|}{$\mathrm{M}+2$} & \multicolumn{2}{|c|}{ M-69 } & \multicolumn{2}{|c|}{$\mathrm{M}+2$} & \multicolumn{2}{|c|}{$\mathrm{M}+30$} \\
\hline & & \multicolumn{2}{|c|}{12.284} & \multicolumn{2}{|c|}{7.123} & \multicolumn{2}{|c|}{9.236} & \multicolumn{2}{|c|}{16.257} & \multicolumn{2}{|c|}{17.477} & \multicolumn{2}{|c|}{18.988} \\
\hline & & $\mathrm{m} / \mathrm{z}$ & int. & $\mathrm{m} / \mathrm{z}$ & int. & $\mathrm{m} / \mathrm{z}$ & int. & $\mathrm{m} / \mathrm{z}$ & int. & $\mathrm{m} / \mathrm{z}$ & int. & $\mathrm{m} / \mathrm{z}$ & int. \\
\hline$[\mathrm{MH}]^{+^{\bullet}}$ & 0 & 304 & 100 & 320 & 98 & 306 & 20 & 235 & 92 & 306 & 75 & 334 & 60 \\
\hline$[\mathrm{M}+\mathrm{Na}]^{+^{\bullet}}$ & +22 & 326 & 5 & 342 & 15 & 328 & 14 & - & - & 328 & 10 & 356 & 70 \\
\hline$\left[\mathrm{MH}-\mathrm{H}_{2} \mathrm{O}\right]^{+^{\bullet}}$ & -18 & 286 & 53 & 302 & 100 & 288 & 25 & 217 & 100 & 288 & 100 & 316 & 70 \\
\hline$[\mathrm{MH}-\mathrm{CO}]^{+^{\bullet}}$ & -28 & - & - & - & - & 278 & 70 & 207 & 70 & - & - & 306 & 55 \\
\hline$\left[\mathrm{MH}-\mathrm{C}_{2} \mathrm{H}_{5} \mathrm{~N}\right]^{+^{\bullet}}$ & -43 & - & - & - & - & - & - & - & - & 263 & 10 & - & - \\
\hline$\left[\mathrm{MH}-\mathrm{CO}_{2}\right]^{{ }^{\bullet}}$ & -44 & 260 & 20 & - & - & - & - & - & - & - & - & - & - \\
\hline$\left[\mathrm{MH}-\mathrm{CO}-\mathrm{NH}_{3}\right]^{+^{\bullet}}$ & -45 & - & - & 275 & 15 & - & - & - & - & - & - & - & - \\
\hline$\left[\mathrm{MH}-\mathrm{H}_{2} \mathrm{O}-\mathrm{CO}\right]^{+}$ & -46 & - & - & - & - & 260 & 65 & 189 & 76 & - & - & 288 & 100 \\
\hline$\left[\mathrm{MH}-\mathrm{H}_{2} \mathrm{O}-\mathrm{C}_{2} \mathrm{H}_{5} \mathrm{~N}\right]^{+}$ & -61 & 243 & 10 & - & - & - & - & - & - & 245 & 5 & - & - \\
\hline$\left[\mathrm{MH}-\mathrm{H}_{2} \mathrm{O}-\mathrm{CO}_{2}\right]^{+^{\bullet}}$ & -62 & - & - & 258 & 98 & 244 & 80 & - & - & - & - & - & - \\
\hline$\left[\mathrm{MH}-2 \mathrm{H}_{2} \mathrm{O}-\mathrm{CO}\right]^{+}$ & -64 & - & - & - & - & - & - & - & - & 242 & 10 & - & - \\
\hline$\left[\mathrm{MH}-\mathrm{CO}_{2}-\mathrm{C}_{2} \mathrm{H}_{5} \mathrm{~N}\right]^{+}$ & -87 & 217 & 72 & - & - & - & - & - & - & - & - & - & - \\
\hline$\left[\mathrm{MH}-\mathrm{H}_{2} \mathrm{O}-\mathrm{CO}-\mathrm{C}_{2} \mathrm{H}_{5} \mathrm{~N}\right]^{{ }^{\circ}}$ & -89 & - & - & - & - & 217 & 100 & - & - & - & - & - & - \\
\hline$\left[\mathrm{MH}-2 \mathrm{H}_{2} \mathrm{O}-2 \mathrm{CO}\right]^{+}$ & -92 & - & - & - & - & - & - & - & - & - & - & 242 & 20 \\
\hline$\left[\mathrm{MH}-\mathrm{H}_{2} \mathrm{O}-2 \mathrm{CO}-\mathrm{C}_{2} \mathrm{H}_{5} \mathrm{~N}\right]^{+^{\bullet}}$ & -117 & - & - & - & - & 189 & 12 & - & - & 189 & 6 & - & - \\
\hline
\end{tabular}

$\mathrm{RT}=\mathrm{LC}$ retention time; int. $=$ MS intensity $(\%)$. 
Table S7. Product distribution (\%) $)^{\mathrm{a}}$ of FQs in reaction with $\mathrm{MnO}_{2}$.

(a) Reaction time $=3.5$ hours:

\begin{tabular}{lcccccc}
\hline & $\mathbf{M H}^{+}$ & $\mathbf{M}$ & $\mathbf{M + 3 0}$ & $\mathbf{M}^{\mathbf{b}}$ & $\mathbf{M}^{\mathbf{b}}$ & Total $^{\mathbf{c}}$ \\
\hline Ciprofloxacin & 332 & 17 & 6 & 1 & 10 & 34 \\
Enrofloxacin & 360 & 19 & 10 & 4 & 10 & 42 \\
Norfloxacin & 320 & 22 & 5 & 2 & 10 & 38 \\
Ofloxacin & 362 & 21 & 23 & $<1$ & 5 & 50 \\
Lomefloxacin $_{\text {Pipemidic Acid }}{ }^{\mathbf{d}}$ & 352 & 45 & 18 & 1 & 15 & 79 \\
\hline
\end{tabular}

(b) Reaction time $=54$ hours:

\begin{tabular}{lcccccc}
\hline & $\mathbf{M H}^{+}$ & $\mathbf{M}$ & $\mathbf{M + 3 0}$ & $\mathbf{M + 2}^{\mathbf{b}}$ & $\mathbf{M - 6 9}$ & Total $^{\mathbf{c}}$ \\
\hline Ciprofloxacin & 332 & 0 & 7 & 3 & 9 & 19 \\
Enrofloxacin & 360 & 0 & 5 & 6 & 9 & 15 \\
Norfloxacin & 320 & 0 & 8 & 2 & 12 & 21 \\
Ofloxacin & 362 & 0 & 27 & $<1$ & 8 & 36 \\
Lomefloxacin $^{\text {Pipemidic Acid }}{ }^{\mathbf{e}}$ & 352 & 3 & 24 & 2 & 13 & 42 \\
\hline
\end{tabular}

Reaction conditions: $[\mathrm{FQ}]_{0}=0.2 \mathrm{mM},\left[\mathrm{MnO}_{2}\right]_{0}=2 \mathrm{mM}, 0.01 \mathrm{M} \mathrm{pH} 5$ acetic acid buffer, $22^{\circ} \mathrm{C}$. ${ }^{\mathrm{a}}$ Each percentage is the ratio of the corresponding product MS area to the parent compound MS area in the control experiment. ${ }^{b}$ The MS areas of the two $\mathrm{M}+2$ products were combined. ${ }^{\mathrm{c}}$ The sum of all detectable areas. ${ }^{\mathrm{d}}$ Reaction time $=20$ hours. ${ }^{\mathrm{e}}$ Reaction time $=44$ hours. 
Table S8. LC-ESI-MS fragments of 1-phenylpiperazine (PP) and its oxidation products.

\begin{tabular}{|c|c|c|c|c|c|c|c|c|c|c|c|}
\hline & & \multicolumn{2}{|c|}{$\begin{array}{l}\mathrm{PP} \\
\mathrm{M}\end{array}$} & \multicolumn{2}{|c|}{$\mathrm{M}+2$} & \multicolumn{2}{|c|}{ M-26 } & \multicolumn{2}{|c|}{$\mathrm{M}+30$} & \multicolumn{2}{|c|}{$2(\mathrm{M}+30)$} \\
\hline \multirow[t]{2}{*}{ RT (min) } & & \multicolumn{2}{|c|}{7.268} & \multicolumn{2}{|c|}{4.076} & \multicolumn{2}{|c|}{5.242} & \multicolumn{2}{|c|}{12.418} & \multicolumn{2}{|c|}{18.408} \\
\hline & & $\mathrm{m} / \mathrm{z}$ & int. & $\mathrm{m} / \mathrm{z}$ & int. & $\mathrm{m} / \mathrm{z}$ & int. & $\mathrm{m} / \mathrm{z}$ & int. & $\mathrm{m} / \mathrm{z}$ & int. \\
\hline$[\mathrm{MH}]^{+}$ & 0 & 163 & 100 & 165 & 23 & 137 & 15 & 193 & 25 & 383 & 68 \\
\hline$[2 \mathrm{M}+\mathrm{Na}]^{+^{\bullet}}$ & - & - & - & 353 & 30 & - & - & - & - & - & - \\
\hline$[\mathrm{M}+\mathrm{Na}]^{+^{\bullet}}$ & +22 & - & - & - & - & - & - & 215 & 50 & 405 & 100 \\
\hline$\left[\mathrm{MH}-\mathrm{NH}_{3}\right]^{{ }^{\bullet}}$ & -17 & - & - & 148 & 100 & 120 & 100 & - & - & - & - \\
\hline$\left[\mathrm{MH}-\mathrm{H}_{2} \mathrm{O}\right]^{+^{\bullet}}$ & -18 & - & - & - & - & - & - & 175 & 20 & - & - \\
\hline$[\mathrm{MH}-\mathrm{CO}]^{+}$ & -28 & - & - & - & - & - & - & 165 & 60 & 355 & 35 \\
\hline$\left[\mathrm{MH}-\mathrm{C}_{2} \mathrm{H}_{5} \mathrm{~N}\right]^{+^{\cdot}}$ & -43 & 120 & 10 & - & - & - & - & - & - & - & - \\
\hline$\left[\mathrm{MH}-\mathrm{CO}-\mathrm{NH}_{3}\right]^{\bullet^{\bullet}}$ & -45 & - & - & 120 & 12 & - & - & 148 & 100 & - & - \\
\hline$\left[\mathrm{MH}-\mathrm{C}_{3} \mathrm{H}_{7} \mathrm{~N}\right]^{+^{\bullet}}$ & -58 & 105 & 8 & - & - & - & - & - & - & - & - \\
\hline$[\mathrm{MH}-2 \mathrm{CO}]^{+^{\bullet}}$ & -56 & - & - & - & - & - & - & 137 & 5 & - & - \\
\hline$\left[\mathrm{MH}-2 \mathrm{CO}-\mathrm{NH}_{3}\right]^{+^{\bullet}}$ & -73 & - & - & - & - & - & - & 120 & 10 & - & - \\
\hline$\left[\mathrm{MH}-2 \mathrm{CO}-\mathrm{CH}_{3}-\mathrm{NH}_{3}\right]^{+^{\bullet}}$ & -88 & - & - & - & - & - & - & 105 & 10 & - & - \\
\hline
\end{tabular}

$\mathrm{RT}=\mathrm{LC}$ retention time; int. $=$ MS intensity $(\%)$ 
Table S9. LC-ESI-MS fragments of 1-phenylmorpholine (PM) and its oxidation products.

\begin{tabular}{|c|c|c|c|c|c|c|c|c|c|c|c|c|c|}
\hline & & \multicolumn{2}{|c|}{$\begin{array}{c}\text { PM } \\
\text { M }\end{array}$} & \multicolumn{2}{|c|}{$\begin{array}{c}\text { Dimeric } \\
\text { product_1 }\end{array}$} & \multicolumn{2}{|c|}{$\begin{array}{c}\text { Dimeric } \\
\text { product_2 }\end{array}$} & \multicolumn{2}{|c|}{$\begin{array}{c}\text { Dimeric } \\
\text { product_3 }\end{array}$} & \multicolumn{2}{|c|}{$\mathrm{M}+2$} & \multicolumn{2}{|c|}{$\mathrm{M}+30$} \\
\hline \multirow[t]{2}{*}{ RT (min) } & & \multicolumn{2}{|c|}{29.142} & \multicolumn{2}{|c|}{3.881} & \multicolumn{2}{|c|}{5.228} & \multicolumn{2}{|c|}{6.626} & \multicolumn{2}{|c|}{12.166} & \multicolumn{2}{|c|}{25.402} \\
\hline & & $\mathrm{m} / \mathrm{z}$ & int. & $\mathrm{m} / \mathrm{z}$ & int. & $\mathrm{m} / \mathrm{z}$ & int. & $\mathrm{m} / \mathrm{z}$ & int. & $\mathrm{m} / \mathrm{z}$ & int. & $\mathrm{m} / \mathrm{z}$ & int. \\
\hline$[\mathrm{MH}]^{+}$ & 0 & 164 & 45 & 327 & 100 & 327 & 10 & 355 & 100 & 166 & 15 & 194 & 3 \\
\hline$[\mathrm{M}+\mathrm{Na}]^{+}$ & +22 & - & - & - & - & - & - & - & - & 188 & 100 & 216 & 100 \\
\hline$\left[\mathrm{MH}-\mathrm{H}_{2} \mathrm{O}\right]^{+}$ & -18 & 146 & 20 & - & - & - & - & - & - & - & - & - & - \\
\hline$\left[\mathrm{MH}-\mathrm{C}_{2} \mathrm{H}_{4} \mathrm{O}\right]^{+}$ & -44 & 120 & 100 & 283 & 10 & 283 & 100 & 311 & 7 & - & - & - & - \\
\hline$\left[\mathrm{MH}-\mathrm{H}_{2} \mathrm{O}-\mathrm{CO}\right]^{+^{\circ}}$ & -46 & - & - & - & - & - & - & - & - & - & - & 148 & 46 \\
\hline$\left[\mathrm{MH}-\mathrm{C}_{3} \mathrm{H}_{7} \mathrm{O}\right]^{+^{\bullet}}$ & -59 & 105 & 10 & - & - & - & - & - & - & - & - & - & - \\
\hline$\left[\mathrm{MH}-\mathrm{C}_{3} \mathrm{H}_{8} \mathrm{O}\right]^{+}$ & -60 & - & - & 267 & 7 & - & - & - & - & - & - & - & - \\
\hline$\left[\mathrm{MH}-\mathrm{C}_{2} \mathrm{H}_{4} \mathrm{O}-\mathrm{H}_{2} \mathrm{O}\right]^{+}$ & -62 & - & - & - & - & 265 & 6 & - & - & - & - & - & - \\
\hline$\left[\mathrm{MH}-\mathrm{C}_{2} \mathrm{H}_{4} \mathrm{O}-\mathrm{CO}\right]^{+}$ & -72 & - & - & - & - & 255 & 25 & - & - & - & - & - & - \\
\hline$\left[\mathrm{MH}-\mathrm{C}_{4} \mathrm{H}_{9} \mathrm{O}\right]^{+}$ & -73 & 91 & 9 & 254 & 6 & - & - & - & - & - & - & - & - \\
\hline$\left[\mathrm{MH}-\mathrm{C}_{2} \mathrm{H}_{4} \mathrm{O}-\mathrm{H}_{2} \mathrm{O}-\mathrm{CO}\right]^{+}$ & -88 & - & - & - & - & 237 & 35 & - & - & - & - & - & - \\
\hline$\left[\mathrm{MH}-2 \mathrm{H}_{2} \mathrm{O}-2 \mathrm{CO}\right]^{+}$ & -92 & - & - & - & - & - & - & 263 & 8 & - & - & - & - \\
\hline$\left[\mathrm{MH}-\mathrm{C}_{2} \mathrm{H}_{4} \mathrm{O}-\mathrm{C}_{4} \mathrm{H}_{9} \mathrm{O}\right]^{{ }^{\circ}}$ & -118 & - & - & - & - & 209 & 30 & - & - & - & - & - & - \\
\hline$\left[\mathrm{MH}-2 \mathrm{C}_{3} \mathrm{H}_{8} \mathrm{O}\right]^{+^{-}}$ & -119 & - & - & 208 & 7 & - & - & - & - & - & - & - & - \\
\hline
\end{tabular}

$\mathrm{RT}=$ retention time; int. $=$ MS intensity $(\%)$. 


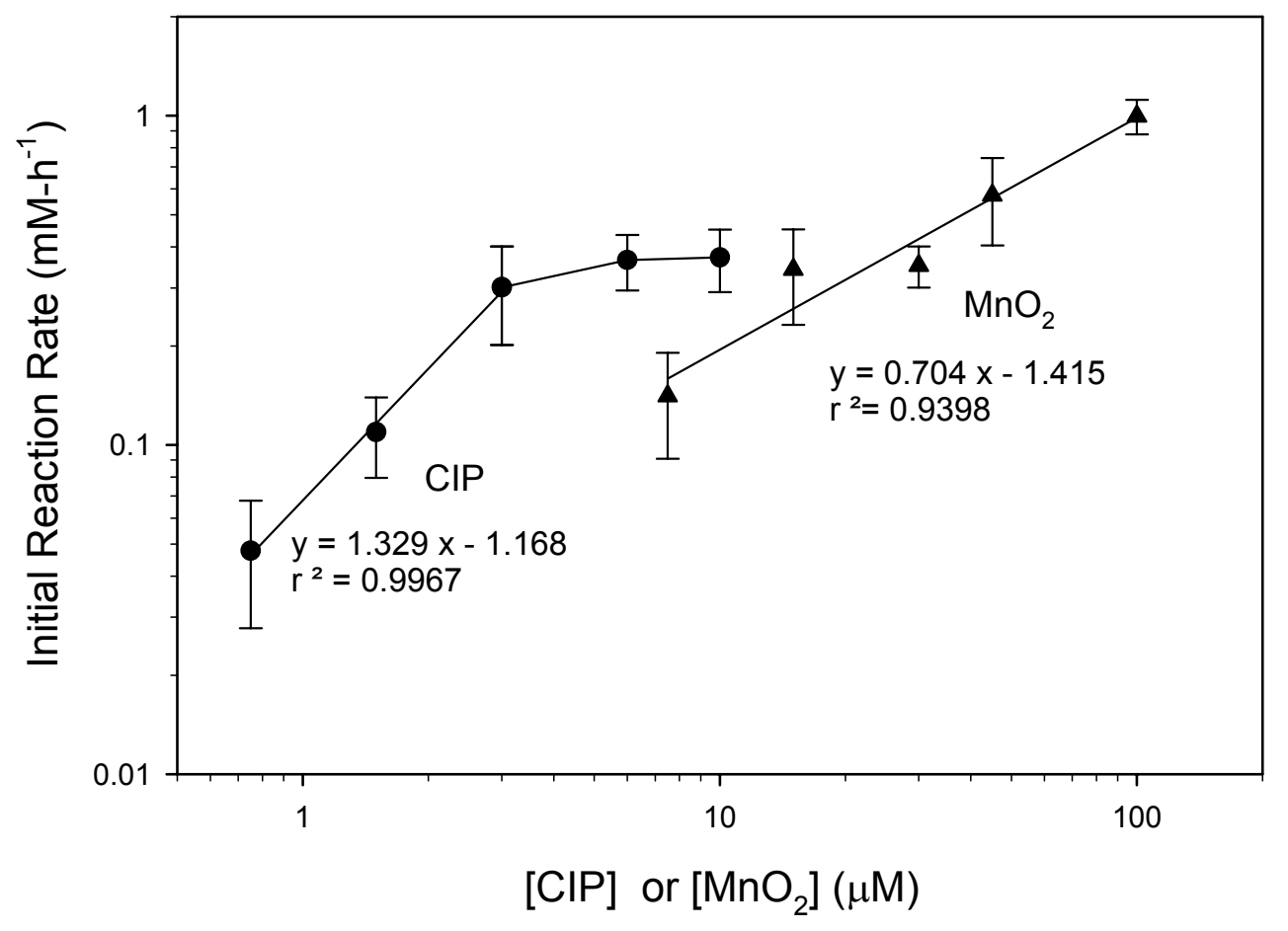

Figure S1. Effect of CIP and $\mathrm{MnO}_{2}$ loadings on the initial reaction rate $\left(\mathrm{pH} 5,22^{\circ} \mathrm{C}\right)$. 


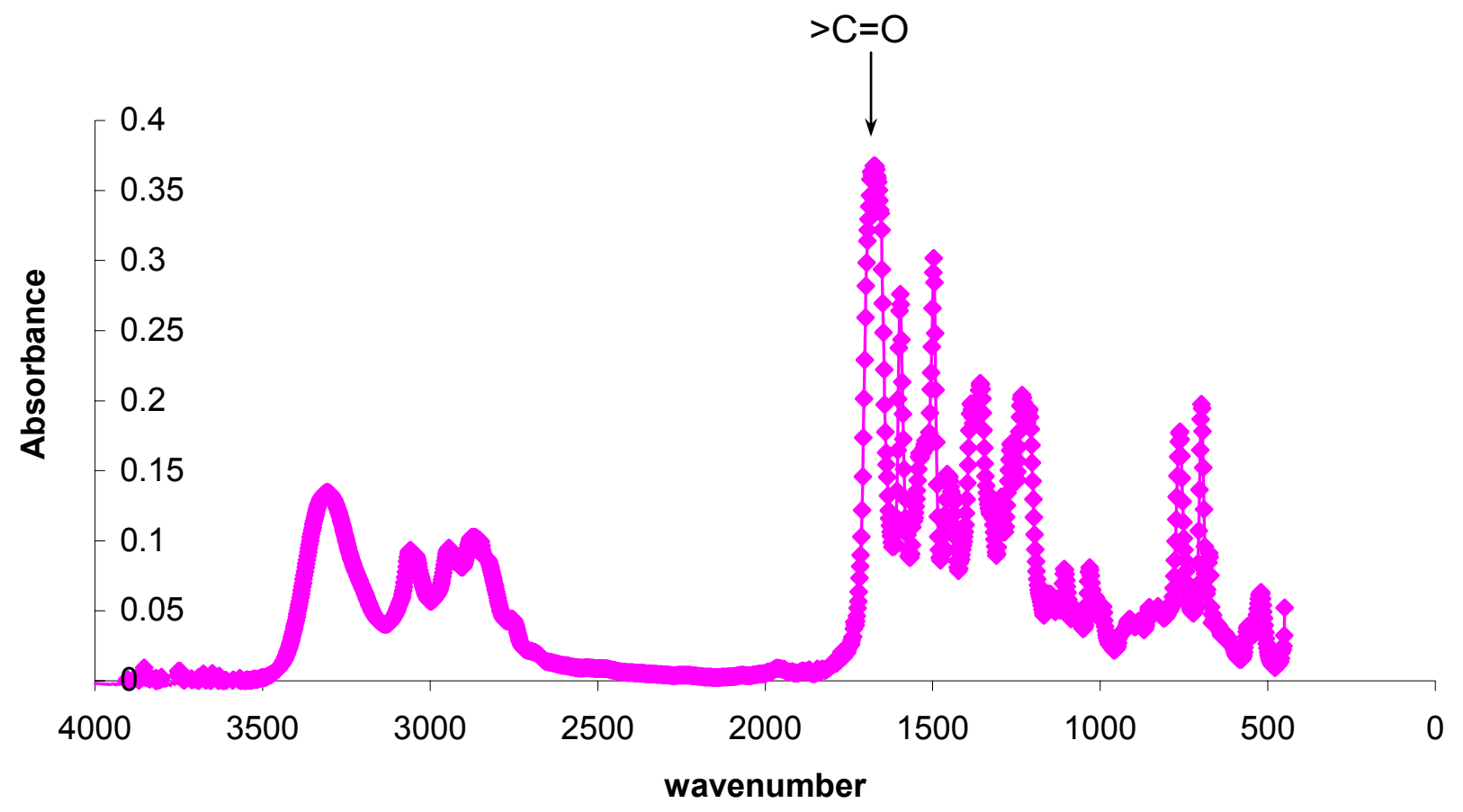

Figure S2. FTIR spectrum of the mixture of PP oxidation products. 